\title{
Automatic Association of Web Directories with Word Senses
}

\author{
Celina Santamaría* \\ UNED, Madrid
}

\author{
Julio Gonzalo* \\ UNED, Madrid
}

\author{
Felisa Verdejo* \\ UNED, Madrid
}

We describe an algorithm that combines lexical information (from WordNet 1.7) with Web directories (from the Open Directory Project) to associate word senses with such directories. Such associations can be used as rich characterizations to acquire sense-tagged corpora automatically, cluster topically related senses, and detect sense specializations. The algorithm is evaluated for the 29 nouns (147 senses) used in the Senseval 2 competition, obtaining 148 (word sense, Web directory) associations covering $88 \%$ of the domain-specific word senses in the test data with $86 \%$ accuracy. The richness of Web directories as sense characterizations is evaluated in a supervised word sense disambiguation task using the Senseval 2 test suite. The results indicate that, when the directory/word sense association is correct, the samples automatically acquired from the Web directories are nearly as valid for training as the original Senseval 2 training instances. The results support our hypothesis that Web directories are a rich source of lexical information: cleaner, more reliable, and more structured than the full Web as a corpus.

\section{Introduction}

Combining the size and diversity of the textual material on the World Wide Web with the power and efficiency of current search engines is an attractive possibility for acquiring lexical information and corpora. A widespread example is spell-checking: Many Web users routinely use search engines to assess which is the "correct" (i.e. with more hits in the Web) spelling of words. Among NLP researchers, Web search engines have already been used as a point of departure for extraction of parallel corpora, automatic acquisition of sense-tagged corpora, and extraction of lexical information.

Extraction of parallel corpora. In Resnik (1999), Nie, Simard, and Foster (2001), Ma and Liberman (1999), and Resnik and Smith (2002), the Web is harvested in search of pages that are available in two languages, with the aim of building parallel corpora for any pair of target languages. This is a very promising technique, as many machine translation (MT) and cross-language information retrieval (CLIR) strategies rely on the existence of parallel corpora, which are still a scarce resource. Such Web-mined parallel corpora have proved to be useful, for instance, in the context of the CLEF (Cross-Language Evaluation Forum) CLIR competition, in which many participants use such parallel corpora (provided by the University of Montreal) to improve the performance of their systems (Peters et al. 2002).

Automatic acquisition of sense-tagged corpora. The description of a word sense can be used to build rich queries in such a way that the occurrences of the word in the documents retrieved are, with some probability, associated with the desired sense. If the probability is high enough, it is then possible to acquire sense-tagged corpora

* ETS Ingeniería Informática de la UNED, c/ Juan del Rosal, 16, Ciudad Universitaria, 28040 Madrid, Spain. E-mail: \{celina,julio,felisa\}@lsi.uned.es 
in a fully automatic fashion. Again, this is an exciting possibility that would solve the current bottleneck of supervised word sense disambiguation (WSD) methods (namely, that sense-tagged corpora are very costly to acquire).

One example of this kind of technique is Mihalcea and Moldovan (1999), in which a precision of $91 \%$ is reported over a set of 20 words with 120 senses. In spite of the high accuracy obtained, such methodology did not perform well in the comparative evaluation reported in Agirre and Martínez (2000), perhaps indicating that examples obtained from the Web may have topical biases (depending on the word), and that further refinement is required. For instance, a technique that behaves well with a small set of words might fail in the common cases in which a new sense is predominant on the Web (e.g., oasis or nirvana as music groups, tiger as a golfer, jaguar as a car brand).

Extraction of lexical information. In Agirre et al. (2000), search engines and the Web are used to assign Web documents to WordNet concepts. The resulting sets of documents are then processed to build topic signatures, that is, sets of words with weights that enrich the description of a concept. In Grefenstette (1999), the number of hits in Web search engines is used as a source of evidence to select optimal translations for multiword expressions. For instance, apple juice is selected as a better translation than apple sap for the German ApfelSaft because apple juice hits a thousand times more documents in AltaVista. Finally, in Joho and Sanderson (2000) and Fujii and Ishikawa (1999), the Web is used as a resource to provide descriptive phrases or definitions for technical terms.

A common problem to all the above applications is how to detect and filter out all the noisy material on the Web, and how to characterize the rest (Kilgarriff 2001b).

Our starting hypotheses is that Web directories (e.g., Yahoo, AltaVista or Google directories, the Open Directory Project [ODP]), in which documents are mostly manually classified in hierarchical topical clusters, are an optimal source for acquiring lexical information; their size is not comparable to the full Web, but they are still enormous sources of semistructured, semifiltered information waiting to be mined.

In this article, we describe an algorithm for assigning Web directories (from the Open Directory Project $\langle\mathrm{http}: / / \mathrm{dmoz} . o r g\rangle$ ) as characterizations for word senses in WordNet 1.7 noun synsets (Miller 1990). For instance, let us consider the noun circuit, which has six senses in WordNet 1.7. These senses are grouped in synsets, together with their synonym terms, and linked to broader (more general) synsets via hypernymy relations:

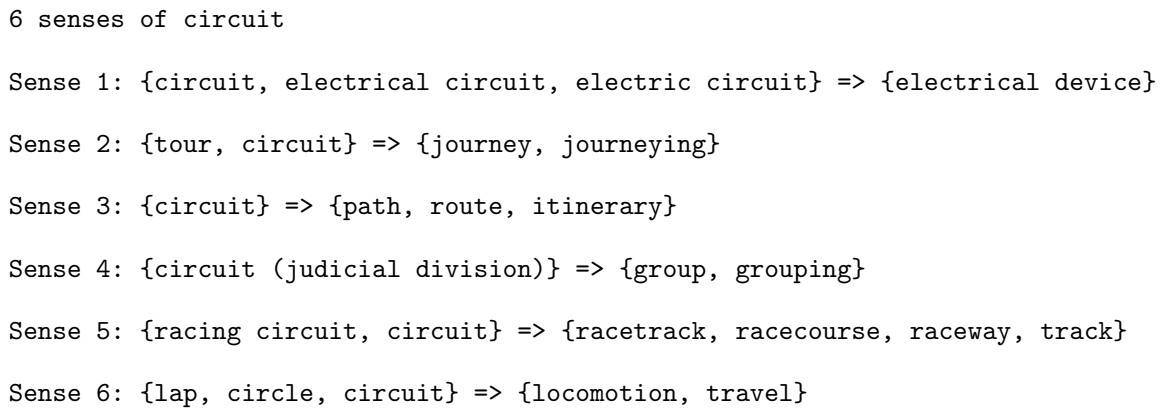

Our algorithm associates circuit 1 (electric circuit) with ODP directories such as 
whereas circuit 5 (racing circuit) is tagged with directories such as

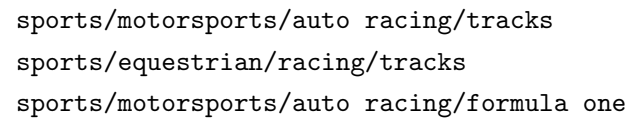

Every ODP directory has an associated URL, which contains a description of the directory and a number of Web sites that have been manually listed as pertaining to the directory topic, accompanied by brief descriptions of each site. This information is completed with a list of subdirectories, each containing more Web sites and subdirectories. Finally, some directories also have pointers to the same category in other languages. For instance, the Web page for the directory sports/motorsports/auto racing/tracks can be seen in Figure 1. This directory contains links and descriptions for 846 Web sites organized in 12 subdirectories, a link to a related directory (sports/motorsports/karting/tracks) and a link to the same category in French.

The association of word senses with Web directories is related to the assignment of domain labels to WordNet synsets as described in Magnini and Cavaglia (2000), in which WordNet is (manually) enriched with domain categories from the Dewey Decimal Classification (DDC). Some clear differences between the two are that directories from the ODP are assigned automatically, are richer and deeper and, more importantly,

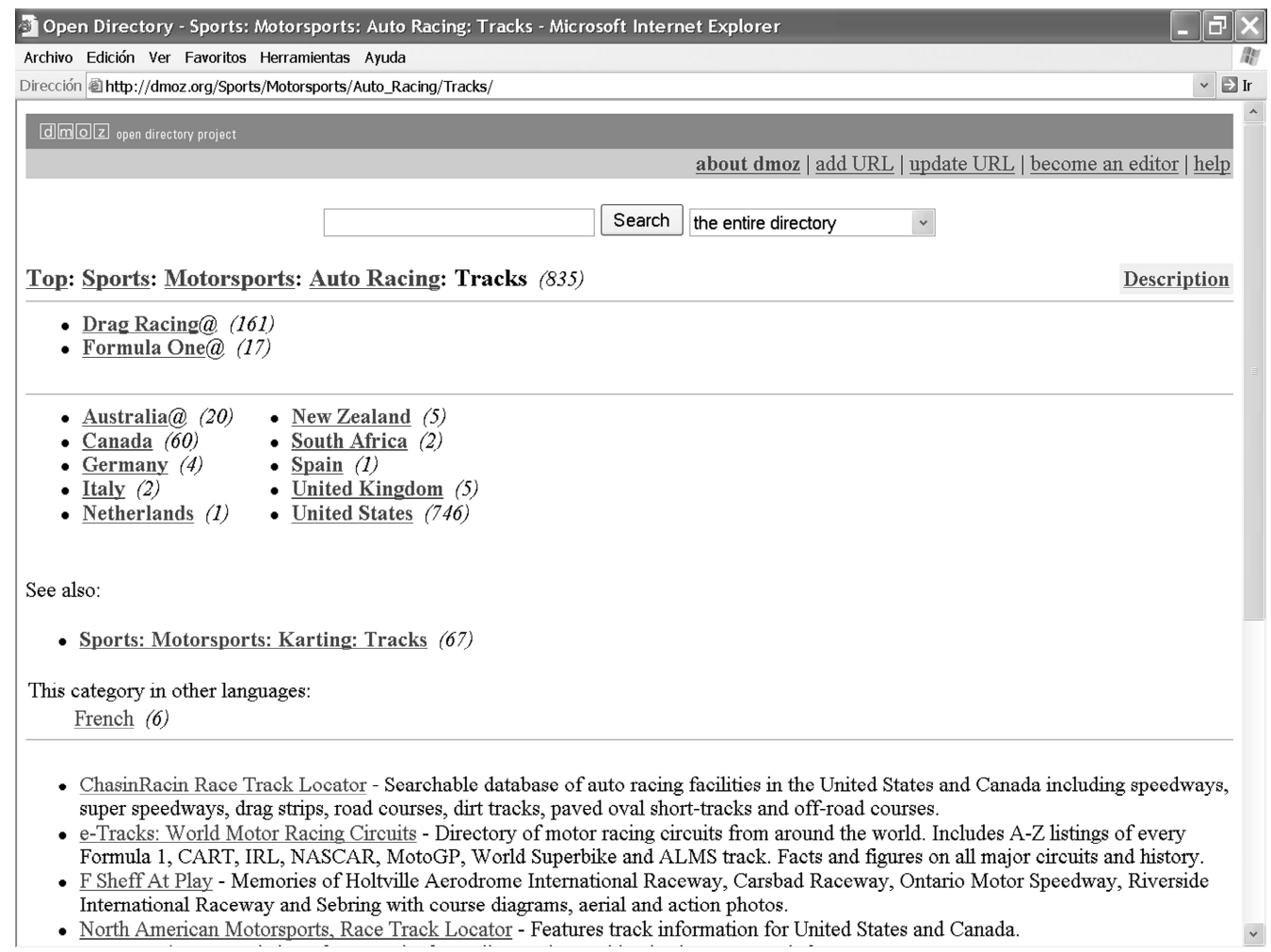

Figure 1

Contents of an ODP Web directory associated with circuit 5 (racing circuit). 
come with a large amount of associated information directly retrievable from the Web. DDC categories, on the other hand, are a stable domain characterization compared to Web directories.

As WordNet and ODP are both hierarchical structures, connecting them is also related to research in mapping thesauruses for digital libraries, ontologies, and data structures in compatible databases. A salient feature of our task is, however, that we do not intend to map both structures, as they are of a quite different nature (lexicalized English concepts versus topics on the Web). Our goal is rather to associate individual items in a many-to-many fashion. A word sense may be characterized with several Web directories, and a Web directory may be suitable for many word senses.

The most direct applications of word sense/Web directory associations are

- Clustering of senses with identical or very similar categories.

- Refinement of senses into specialized variants (e.g., equestrian circuit and formula one circuit as specializations of racing circuit in the example above).

- Extraction of sense-tagged corpora from the Web sites listed under the appropriate directories.

In Section 2 we describe the proposed algorithm. In Section 3, we evaluate the precision and recall of the algorithm for the set of nouns used in the Senseval 2 WSD competition. In Section 4, we make a preliminary experiment using the material from ODP directories as training corpora for a supervised WSD system. In section 5, we present the results of applying the algorithm to most WordNet 1.7 nouns. Finally, in Section 6 we draw some conclusions.

\section{Algorithm}

Overall, the system takes a WordNet 1.7 noun as input, generates and submits a set of queries into the ODP, filters the information obtained from the search engine, and returns a set of ODP directories classified as (1) pseudo-domain labels for some word sense, (2) noise, and (3) salient noise (i.e., directories that are not suitable for any sense in WordNet but could reveal and characterize a new relevant sense of the noun). In case (1), the WordNet sense $\leftrightarrow$ ODP directory association also receives a probability score. A detailed description of the algorithm steps follows.

\subsection{Querying ODP Structure}

For every sense $w_{i}$ of the noun $w$, a query $q_{i}$ is generated, including $w$ as compulsory term, the synonyms and direct hypernyms of $w_{i}$ as optional terms, and the synonyms of other senses of $w$ as negated (forbidden) terms. These queries are submitted to ODP, and a set of directories is retrieved. For instance, for circuit, the following queries are generated and sent to the ODP search engine: ${ }^{1}$

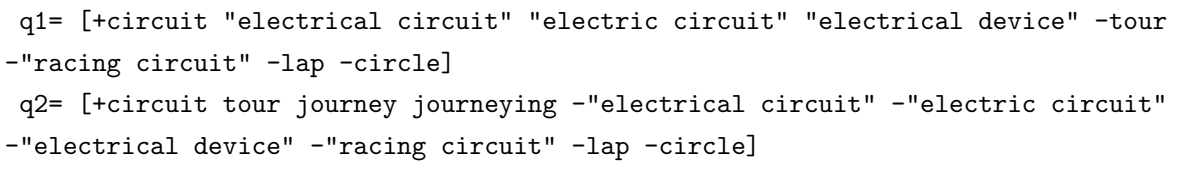

1 In ODP queries, compulsory terms are denoted by + and forbidden terms by - . 


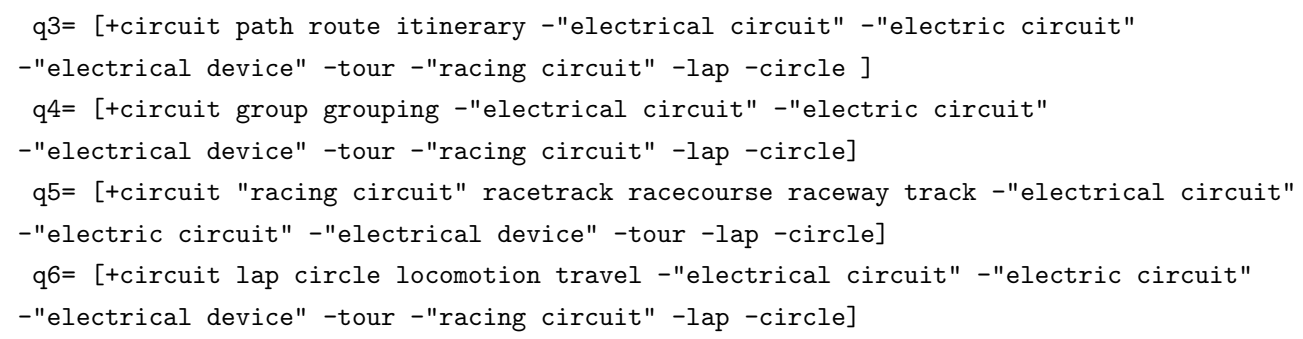

\subsection{Representing Retrieved Directory Descriptions}

For every directory $d$, a list of words $l(d)$ is obtained removing stopwords and preserving all content words in the directory path. For instance, one of the directories produced by the circuit queries is

$$
d=\text { business/industries/electronics and electrical/contract manufacturers }
$$

which is characterized by the following word list:

$$
l(d)=\text { [business, industries, electronics, electrical, contract, manufacturers] }
$$

\subsection{Representing WordNet Senses}

For every sense $w_{j}$, a list $l\left(w_{j}\right)$ of words is made with

- all nouns in the hypernym chain of maximal length 6

- all hyponyms

- all meronyms, holonyms, and coordinate terms

of $w_{j}$ in WordNet. $l\left(w_{j}\right)$ is used as a description of the sense $w_{j}$. For instance, circuit 1 receives the following description:

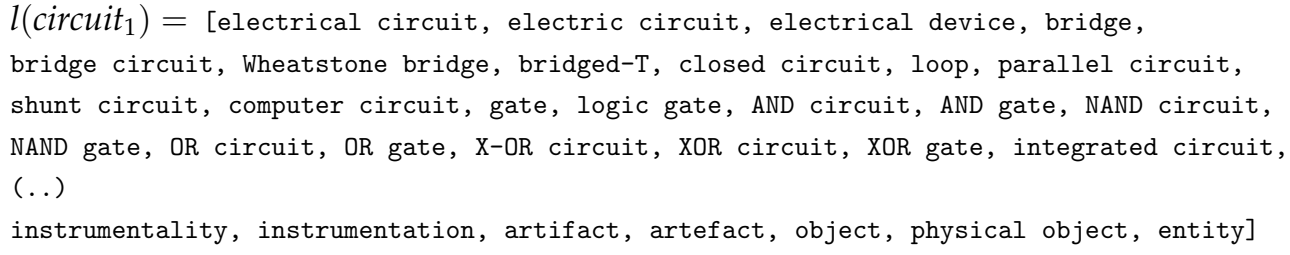

\subsection{Sense/Directory Comparisons}

For every sense description $l\left(w_{j}\right)$, a comparison is made with the terms in the directory description $l(d)$. This comparison is based on the hypothesis that the terms in an appropriate directory for a word sense will have some correlation with the sense description via WordNet semantic relations. In other words, our assumption is that the path to the directory in the ODP topical structure will have some degree of overlapping with the hyponymy path to the word sense in the WordNet hierarchical structure.

For this comparison, we simply count the number of co-occurrences between words in $l\left(w_{j}\right)$ and words in $l(d)$. Repeated terms are not discarded, as repetition is correlated with stronger associations. Other, better-grounded comparisons, such as the cosine between $l\left(w_{j}\right)$ and $l(d)$, were empirically discarded because of the small size and small amount of overlapping of the average vectors. 


\subsection{Candidate Sense/Directory Associations}

The association vector $v(d, w)$ has as many components as senses for $w$ in WordNet 1.7; the $i$ th component, $v(d, w)_{i}$ represents the number of matches between the directory $l(d)$ and the sense descriptor $l\left(w_{j}\right)$. For instance, the association vector of

business/industries/electronics and electrical/contract manufacturers

with circuit is

$$
v(d, \text { circuit })=(6,0,0,0,0,0)
$$

that is, six coincidences for sense 1 (the electric circuit sense), which has the associated vector shown in the previous section (which includes five occurrences of electrical and one occurrence of electronic). The rest of the sense descriptions have no coincidences with the directory description.

$v(d, w)$ is the basis for making candidate assignments of suitable senses for directory $d$ : If one of the components $v(d, w)_{j}$ is not null, we assign the sense $w_{j}$ to the directory $d$. If all components are null, the directory is provisionally classified as noise or new sense. If more than one component is not null, the senses $i$ with maximal $v(d, w)_{i}$ are all considered candidates. These candidate assignments are confirmed or discarded after passing a number of filters and receiving a confidence score $C\left(d, w_{j}\right)$, both of which are described below.

\subsection{Filters}

Filters are simple heuristics that contribute to a more accurate classification of the relations predicted by the co-ocurrence vector $v(d, w)$. We are currently using two filters: One differentiates nouns and noun modifiers to prevent wrong associations, and another detects sense specializations.

2.6.1 Modifiers. Frequently, the ODP search engine retrieves directories in which the noun to be searched, $w$, has as a noun modifier role. Such cases usually produce erroneous associations. For instance, the directory

library/sciences/animals \& wildlife/mammals/tamarins/golden lion tamarin

is erroneously associated with the mammal sense of lion, which is here a modifier for tamarin.

Modifiers are detected with a set of simple patterns, as the syntactic properties of descriptions in directories are quite simple. In particular, we discard most cases using the structure of the ODP hierarchy, as in this case. The filter analyzes the structure of the directory, detects that the parent category of golden lion tamarin is tamarin, therefore assumes that golden lion tamarin is a specialization of tamarin, and assigns the directory to a suitable sense of tamarin (tamarin 1 in WordNet).

An additional filter (weaker than the previous one) discards compounds according to the position (the searched noun precedes another noun), as in

personal/kids/arts \& entertainment/movies/animals/lion king

This directory could be associated with lion 1 because it contains the word animal, but the assignment is rejected because of the modifier filter. In general, on such occasions the searched noun plays a modifier role (as adjective or noun); discarding all such cases favors precision over recall. In this case, the label is classified as noise. 
2.6.2 Sense Specializations (Hyponyms). A retrieved directory might be appropriate as a characterization of a sense specialization for some of the word senses being considered; our algorithm tries to detect such cases, creating a hyponym of the sense and characterizing the directory with the hyponym.

The filter identifies a directory as a candidate hyponym if it contains explicitly a modifier w pattern (where $w$ is the noun being searched). This filter detects explicit specializations, such as office chair as a hyponym of chair 1, or fox family channel as a hyponym of channel 7, but fails to identify, for instance, memorial day as a hyponym of holiday.

If the candidate hyponym, as a compound, is not present in WordNet, then it is incorporated and described with the directory. If it is already present in WordNet, an additional checking of the hyponymy relation is made. For instance, the directory

business/industries/electronics and electrical/components/integrated circuits

is assigned to the WordNet entry integrated circuit, because integrated circuit is already a hyponym of circuit in WordNet.

\subsection{Confidence Score}

Finally, a confidence score $C\left(d, w_{j}\right)$ for every potential association $\left(d, w_{j}\right)$ is calculated using four empirical criteria:

1. Checking whether $d$ was directly retrieved for the query associated to $w_{j}$.

2. Checking whether the system associates $d$ with one or more senses of the word $w$.

3. Checking the number of coincidences between $l(d)$ and $l\left(w_{j}\right)$.

4. Comparing the previous number with the number of coincidences between $l(d)$ and the other sense descriptions $\left\{l(w)_{i}, i \neq j\right\}$.

The confidence score is a linear combination of these factors, weighted according to an empirical estimation of their relevance:

$$
C\left(d, w_{j}\right)=\sum_{i=1}^{4} \alpha_{i} C_{i}\left(d, w_{j}\right)
$$

where

$$
\begin{gathered}
C_{1}\left(d, w_{j}\right)= \begin{cases}1, & \text { if query }\left(w_{j}\right) \text { retrieves } d \\
0, & \text { otherwise }\end{cases} \\
C_{2}\left(d, w_{j}\right)=1-\frac{k}{n} \\
C_{3}\left(d, w_{j}\right)=\left\{\begin{array}{rr}
1, & \text { if } v_{j} \geq 5 \\
\left(v_{j}+5\right) / 10, & \text { if } \begin{array}{r}
<v_{j} \leq 4 \\
0.5, \quad \text { if } v_{j}=1
\end{array}
\end{array}\right. \\
C_{4}\left(d, w_{j}\right)=\frac{v_{j}-\max _{i \neq j}\left(v_{i}\right),}{\sum_{i=1}^{n} v_{i}}
\end{gathered}
$$

where $v$ is the association vector $v(d, w), n$ the number of senses, $k$ the number of senses for which $v_{j}$ is non-null, and $\alpha_{i}$ are coefficients empirically adjusted to $\left(\alpha_{1}, \alpha_{2}, \alpha_{3}, \alpha_{4}\right)=$ 
$(0.1,0.15,0.4,0.35)$. The value of $C\left(d, w_{j}\right)$ ranges between 0 and 1 (all $C_{i}$ range between 0 and 1 , and the sum of the linear coefficients $\alpha_{i}$ is 1 ). Note that $C_{2}$ cannot reach 1 (but can get asymptotically close to 1 ), and note also that $C_{4}$ cannot take negative values, because, as $\left(d, w_{j}\right)$ is a candidate association, $v_{j}$ is maximal in $v(d, w)$, and therefore $v_{j}-\max _{i \neq j}\left(v_{i}\right)$ ranges between 0 and $v_{j}$.

Let us see an example of how this confidence measure works, calculating $C\left(d, w_{j}\right)$ for the directory

$$
d=\text { business/industries/electronics and electrical/contract manufacturers }
$$

with circuit 1 (electric circuit):

- $C_{1}$. This directory has been retrieved from the query

q1= [+circuit "electrical circuit" "electric circuit" "electrical device"

-tour -"racing circuit" -lap -circle]

corresponding to circuit 1 , which agrees with the association made by the system. Hence $C_{1}=1$.

- $\quad C_{2}$. The association vector $v(d, w)=(6,0,0,0,0,0)$ presents only one non-null coordinate; therefore $C_{2}=1-\frac{1}{6}=0.83$. Note that, in general, this factor prevents $C$ from reaching the upper bound 1 .

- $C_{3}$. As $v_{1}=6, C_{3}=1$. This factor increases along with the number of coincidences between the sense and directory characterizations.

- $\quad C_{4}$. As all other components of $v$ are null, the highest value of the components different from sense 1 is also null $\left(\max _{i \neq j}\left(v_{i}\right)=0\right)$; therefore, $C_{4}=1$. This factor measures the strength of the association $\left(d, w_{1}\right)$ compared with the other possibilities. It decreases when $v(d, w)$ includes more than one non-null coordinate, and their values are similar.

- C. Finally, applying the $\alpha_{i}$ coefficients, we obtain $C(d$, circuit 1$)=0.975$.

The confidence score can be used to set a threshold for accepting/discarding associations. A higher threshold should produce a lower number of highly precise associations; a lower threshold would produce more associations with less accuracy. For the evaluation below, we have retained all directories, regardless of their confidence score, in order to assess how well this empirical measure correlates with correct and useful assignments.

An example of the results produced by the algorithm can be seen in Table 1 . The system assigns directories to senses 1,2, and 5 of circuit (six, two, and three directories, respectively). Some of them are shown in the table, together with a sense specialization, integrated circuit, for sense 1 (electrical circuit). Senses 3, 4, and 6, which did not receive any directory association, do not appear to have domain specificity, but are instead general terms.

\section{Evaluation}

We have analyzed the results of the algorithm for the set of nouns in the Senseval 2 WSD English lexical sample test bed (Kilgarriff 2001a). The Senseval campaigns (Edmonds and Cotton 2001; Kilgarriff and Palmer 2000) are devoted to the comparative evaluation of word sense disambiguation systems in many languages. In the Senseval 2 lexical sample task, a large number of instances (occurrences in context extracted 
Table 1

Results of the association algorithm for circuit.

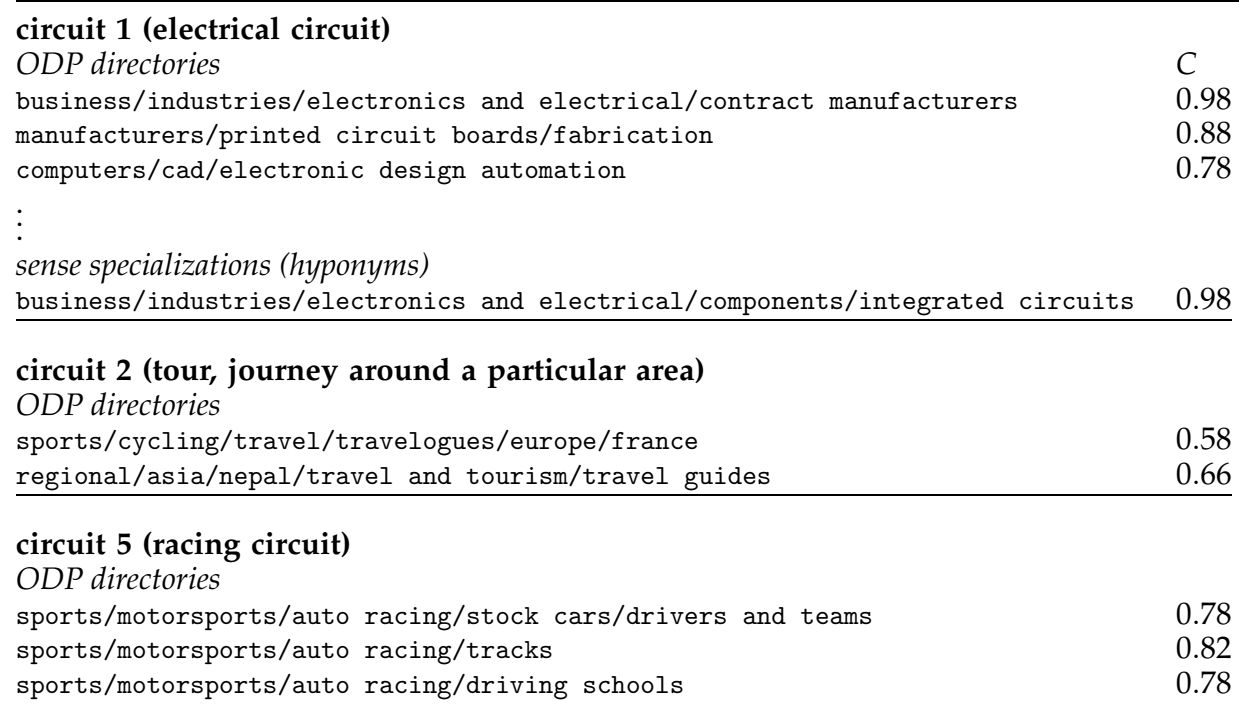

from corpora) for a fixed set of words had to be tagged with the appropriate sense by the participating WSD systems. For English, the sense inventory was a prerelease of WordNet 1.7, and two sets of manually tagged instances were made available: A first set was intended for training supervised systems, and a second set for evaluation of all systems attempting the task. Altogether, the Senseval 2 lexical sample test bed is one of the most widely used resources for studying and comparing word sense disambiguation approaches.

For our evaluation, we have considered the fraction of the Senseval 2 test bed that deals with English nouns: 29 polysemous nouns with a total of 147 word senses. We have applied the algorithm to this set of nouns and examined the results in terms of coverage and quality of the sense/directory associations. Coverage measures how many senses can be characterized with directories, assuming that every domainspecific sense should receive at least one directory. Quality is measured in terms of precision (are the assignments correct?), relevance (are the assignments useful?), and confidence (does the confidence score correlate well with precision and relevance of the associations?).

\subsection{Coverage}

Table 2 shows the 148 directories retrieved by our algorithm, an average of 1.0 directories per sense. The directories, however, are not evenly distributed among senses, covering only 43 different senses with unique directories and 28 specialized (hyponym) senses. In addition, 9 senses are identified as part of potential clusters (i.e., having nonunique directories).

In order to measure the real coverage of the system, we have to estimate how many word senses in the Senseval 2 sample are susceptible to receiving a domain label. For instance, sense in common sense is not associated with any particular topic or domain, whereas sense in word sense can be associated with linguistics or languagerelated topics.

The decision as to whether or not a word sense might receive a domain label is not always a simple, binary one. Hence we have manually tagged all word senses 
Table 2

Coverage of nouns in the Senseval 2 test bed.

\begin{tabular}{|c|c|c|c|c|}
\hline $\begin{array}{l}\text { Senseval } 2 \\
\text { Nouns }\end{array}$ & $\begin{array}{l}\text { Number of } \\
\text { Senses }\end{array}$ & $\begin{array}{l}\text { Number of } \\
\text { Directories }\end{array}$ & $\begin{array}{c}\text { Number of Labeled } \\
\text { Senses }\end{array}$ & $\begin{array}{l}\text { Number of } \\
\text { Hyponyms }\end{array}$ \\
\hline art & 4 & 6 & 1 & 1 \\
\hline authority & 7 & 4 & 2 & 1 \\
\hline bar & 13 & 3 & 3 & 0 \\
\hline bum & 4 & 0 & 0 & 0 \\
\hline chair & 4 & 4 & 1 & 0 \\
\hline channel & 7 & 5 & 1 & 1 \\
\hline child & 4 & 12 & 2 & 0 \\
\hline church & 3 & 24 & 2 & 4 \\
\hline circuit & 6 & 11 & 3 & 1 \\
\hline day & 10 & 15 & 1 & 14 \\
\hline detention & 2 & 1 & 1 & 0 \\
\hline dyke & 2 & 1 & 1 & 0 \\
\hline facility & 5 & 10 & 3 & 0 \\
\hline fatigue & 4 & 0 & 0 & 0 \\
\hline feeling & 6 & 2 & 1 & 0 \\
\hline grip & 7 & 3 & 2 & 0 \\
\hline hearth & 3 & 5 & 2 & 0 \\
\hline holiday & 2 & 2 & 2 & 0 \\
\hline lady & 3 & 0 & 0 & 0 \\
\hline material & 5 & 9 & 2 & 3 \\
\hline mouth & 8 & 0 & 0 & 0 \\
\hline nation & 4 & 4 & 1 & 1 \\
\hline nature & 5 & 0 & 0 & 0 \\
\hline post & 8 & 14 & 5 & 0 \\
\hline restraint & 6 & 4 & 3 & 0 \\
\hline sense & 5 & 0 & 0 & 0 \\
\hline spade & 3 & 3 & 1 & 1 \\
\hline stress & 5 & 5 & 2 & 1 \\
\hline yew & 2 & 1 & 1 & 0 \\
\hline Total & 147 & 148 & 43 & 28 \\
\hline
\end{tabular}

with two criteria (with each tagging performed by a different human annotator): a strict one (only word senses that can clearly receive a domain label are marked as positive) and a loose one (only word senses that are completely generic are marked as negative). The strict judgment gave 59 domain-specific senses in the sample; the loose judgment gave 71 .

With these manual judgments, the coverage of the algorithm is between $73 \%$ (loose judgment) and $88 \%$ (strict judgment). This coverage can be increased by

- Propagating a directory/word sense association to all members of the WordNet synset to which the word sense belongs.

- Propagating directories via hyponymy chains, as in Magnini and Cavaglia (2000).

\subsection{Quality}

We have used three criteria to evaluate the directory/sense associations produced:

- Precision. Is the directory correct (suitable) for the word sense or not? 
- Relevance. Is the directory useful for characterizing the word sense?

- Confidence. How well is the confidence value $C\left(d, w_{j}\right)$ correlated with the precision and relevance of the associations?

3.2.1 Precision. An assignment $\left(d, w_{j}\right)$ is considered correct ( $d$ is suitable for $\left.w_{j}\right)$ unless

1. $\quad d$ adjusts better to some other sense $w_{i}$. For instance, the association of

regional/north america/united states/government/agencies/independent/

federal labor relations authority

as a hyponym of

authority 4 : assurance, self-assurance, confidence, self-confidence, authority, sureness

is considered an error, as the directory would be better suited for a hyponym of sense 5 (authority as administrative unit).

2. The terms in $l(d)$ are contradictory to the definition of the word sense or are better suited for a sense that is not listed in the dictionary. This is the case of

$$
\text { arts/music/bands and artists/offspring }
$$

which is erroneously assigned to child 2: human offspring of any age.

The results of this manual evaluation can be seen in Table 3. The overall precision is $86 \%$.

Regarding potential topical clusters (directories associated with more than one sense of the same word), these are considered correct if (1) the associated directory is correct for all the senses in the cluster and (2) the occurrences of the word on the Web page associated with the directory can be loosely assigned to any of the cluster senses. Twelve out of the 13 clusters extracted are correct according to this criterion.

3.2.2 Confidence Measures. Table 4 shows the distribution of directories according to the confidence measure. Eighty-four percent of the directories have a confidence $C$ over 0.7 , and $41 \%$ over 0.8 . This skewed distribution is consistent with the algorithm filters, which are designed to favor precision rather than recall.

Table 5 shows the distribution of errors in levels of confidence. The percentage of errors in directories with a confidence level below .6 is $25 \%$. This error percentage

Table 3

Precision over Senseval 2 nouns.

\begin{tabular}{lccc}
\hline $\begin{array}{l}\text { Directories Associated } \\
\text { with WordNet Senses }\end{array}$ & $\begin{array}{c}\text { Number of } \\
\text { Directories }\end{array}$ & Number of Correct & Number of Errors \\
\hline Unique sense & 148 & 127 & 21 \\
Potential clustering & 13 & 12 & 1 \\
Total & 161 & $139(86 \%)$ & $22(14 \%)$
\end{tabular}


Table 4

Confidence distribution.

\begin{tabular}{lccc}
\hline Confidence & $C \leq 0.7$ & $0.7<C \leq 0.8$ & $0.8<C$ \\
\hline Number of directories & $\mathbf{2 4}$ & $\mathbf{6 3}$ & $\mathbf{6 1}$ \\
\hline
\end{tabular}

Table 5

Correlation between confidence and correctness.

\begin{tabular}{lcc}
\hline Confidence & Number of Directories & Percentage of Errors \\
\hline$C \leq 0.7$ & 24 & $25 \%$ \\
$0.7<C \leq 0.8$ & 63 & $19 \%$ \\
$C>0.8$ & 61 & $5 \%$ \\
Total & 148 & $14 \%$
\end{tabular}

decreases with increasing levels of confidence, down to $5 \%$ for associations with $C$ over .8. Table 5 indicates that the confidence value, which is assigned heuristically, is indeed correlated with precision.

3.2.3 Relevance. Besides correctness of the associations, we want to measure the usefulness of the directories: How well can they be used to characterize the associated word senses? How much information do they provide about the word senses?

We have performed a manual, qualitative classification of the directories extracted as irrelevant, mildly relevant, or very relevant. An irrelevant directory is compatible with the word sense but does not provide any useful characterization; a mildly relevant directory illustrates the word sense, but not centrally or in some particular aspect or domain. A very relevant directory provides a rich characterization per se and can be considered a domain label for the word sense.

An example of a very relevant directory is

business/industries/electronics and electrical/components/integrated circuit

associated as hyponym of circuit 1 (electrical circuit) with a confidence of $98 \%$. An example of mildly relevant association is

regional/north america/united states/texas/../society and culture/religion

associated with church 1 (Christian church) with a $73 \%$ confidence. Obviously, Texas is not correlated with church, but the directory contains a lot of material (for instance, the Web page of the Northcrest Community Church and many others) that might be used, for instance, to acquire topical signatures for the concept. Hence the mildly relevant judgment. Finally, an example of an irrelevant association is

regional/north america/united states/new york/localities/utica

associated with art 1 (fine art) with a confidence of $66 \%$ (the directory contains a section on Arts at Utica, which would be considered mildly relevant if pointed to explicitly by the label). For the purposes of measuring relevance, all the directories that were judged as incorrect are counted as irrelevant. 
Table 6

Relevance of the directories in the test set.

\begin{tabular}{lccc}
\hline Relevance & Irrelevant & $\begin{array}{c}\text { Mildly } \\
\text { Relevant }\end{array}$ & $\begin{array}{c}\text { Highly } \\
\text { Relevant }\end{array}$ \\
\hline$C \leq 0.7$ & 7 & 4 & 13 \\
$0.7<C \leq 0.8$ & 13 & 12 & 38 \\
$0.8<C$ & 3 & 9 & 49 \\
Total & $23(15 \%)$ & $25(17 \%)$ & $100(67 \%)$
\end{tabular}

The overall relevance figures, and the correlation of relevance with the confidence value, can be seen in Table 6. Sixty-seven percent of the directories are highly relevant to characterize word senses, which is an encouraging result. Also, the set of irrelevant directories $(15 \%)$ is almost identical to the set of erroneous directories (with just one addition), indicating that (almost) all directories that are correct can be used to characterize word senses to some extent.

\section{Example Application: Automatic Acquisition of Sense-Tagged Corpora}

Each ODP directory contains links to related subdirectories and to a large number of Web sites that have been manually classified there. Every link to a Web site includes the name of the site and a short description. For instance, under

business/industries/electronics and electrical/components/integrated circuit

we find over 30 descriptions, such as "Multilink Technology corporation: Manufacture of integrated circuits, modules, and boards for use in both data and telecommunications". In order to perform a first experiment on extraction of sense-tagged corpora, we have used only such descriptions (without exploring the associated Web sites) to build a sense-tagged corpus for Senseval 2 nouns.

Notice that we are not using the contents of the Web sites that belong to a directory, but only the manually added descriptions of Web sites in the directory. Using the Web sites themselves is also an attractive possibility that would produce a much larger corpus at the expense of lower precision.

The extraction is straightforward: When a word sense $w_{i}$ has an associated directory $d$, we scan the site descriptions on the ODP page that corresponds to the directory $d$ and extract all contexts in which $w$ occurs, assuming that in all of them $w$ is used in the sense $i$. Some examples of the training material for circuit can be seen in Table 7 . On average, these examples are shorter than Senseval 2 training instances.

The goal is to compare the performance of a supervised word sense disambiguation system using Senseval 2 training data (hand made for the competition) to that using the sense-tagged corpus from ODP (automatically extracted). We have chosen the Duluth system (Pedersen 2001) to perform the comparison. The Duluth system is a freely available supervised WSD system that participated in the Senseval 2 competition. As we are not concerned with absolute performance, we simply adopted the first of the many available versions of the system (Duluth 1 ).

An obstacle to performing such comparative evaluation is that, as expected, our algorithm assigns ODP directories only to a fraction of all word senses, partly because not every sense is domain-specific, and partly because of lack of coverage. In order to 
Table 7

Examples of training material for circuit.

circuit 1 (electrical circuit)

Electromechanical products for brand name firms; offers printed circuit boards (..)

Offers surface mount, thru-hole, and flex circuit assembly, in circuit and functional (..)

circuit 2 (tour, journey around a particular area)

The Tour du Mont-Blanc is a circuit of $322 \mathrm{~km}$ based in the northern French Alps.

A virtual tour of the circuit by Raimon Bach.

circuit 5 (racing circuit)

The Circuit is a smooth 536 yards of racing for Hot Rod and Stock Car's at the East of (..)

(..) History of the circuit and its banked track and news of Formula 1 (..)

circumvent this problem, we have considered only the subset of 10 Senseval nouns for which our system tags at least two senses: bar, child, circuit, facility, grip, holiday, material, post, restraint, and stress. We have then projected the Senseval 2 training corpus, and the test material, onto the annotations for the word senses already in our ODP-based material. Hence we will evaluate the quality of the training material obtained from Web directories, not the coverage of the approach.

Table 8 shows the training material obtained for that subset of Senseval 2 nouns. A total of 66 directories are used as a source of training instances, of which $17 \%$ are incorrect and will presumably incorporate noise into the training. Table 9 compares the training material for the word senses in this sample, and the results of the supervised WSD algorithm with the Senseval and the ODP training instances.

We have measured the performance of the system in terms of Senseval recall: the number of correctly disambiguated instances over the total number of test instances. Overall, using the Senseval training set gives .73 recall, and training with the automatically extracted ODP instances gives .58 (21\% worse). A decrease of $21 \%$ is significant but nevertheless encouraging, because the Senseval training set is the gold standard for the Senseval test set: It is larger than the ODP set (773 versus 547 instances in this subset), well balanced, built with redundant manual annotations, and part of the same corpus as the test set.

Table 8

Training material obtained for the WSD experiment.

\begin{tabular}{lrrr}
\hline $\begin{array}{l}\text { Word } \\
\text { Senses }\end{array}$ & $\begin{array}{c}\text { Number of Directories } \\
\text { per Sense }\end{array}$ & $\begin{array}{c}\text { Number of Incorrect } \\
\text { Directories }\end{array}$ & $\begin{array}{c}\text { Number of Training } \\
\text { Instances }\end{array}$ \\
\hline bar 1,10 & 1,1 & 0,0 & 1,1 \\
child 1,2 & 3,9 & 0,0 & 3,80 \\
circuit 1,2,5 & $6,2,3$ & $0,0,0$ & $229,2,5$ \\
facility 1,4 & 4,5 & 0,0 & 4,18 \\
grip 2,7 & 2,1 & 0,1 & 17,6 \\
holiday 1,2 & 1,1 & 0,1 & 5,17 \\
material 1,4 & 6,3 & 2,1 & 63,10 \\
post 2,3,4,7,8 & $1,5,1,4,3$ & $1,1,1,0,3$ & $2,7,1,9,3$ \\
restraint 1,4,6 & $2,1,1$ & $0,0,0$ & $2,2,2$ \\
stress 1,2 & 1,4 & 0,0 & 8,50 \\
Total & 66 & 11 & 547
\end{tabular}


Table 9

Results of supervised WSD.

\begin{tabular}{|c|c|c|c|c|c|}
\hline $\begin{array}{l}\text { Word } \\
\text { Senses }\end{array}$ & $\begin{array}{c}\text { Number of } \\
\text { instances } \\
\text { Senseval } \\
\text { Training }\end{array}$ & $\begin{array}{c}\text { Number of } \\
\text { instances } \\
\text { ODP } \\
\text { Training }\end{array}$ & $\begin{array}{c}\text { Number of Test } \\
\text { Instances }\end{array}$ & $\begin{array}{c}\text { Recall } \\
\text { Senseval } \\
\text { Training }\end{array}$ & $\begin{array}{c}\text { Recall } \\
\text { ODP } \\
\text { Training }\end{array}$ \\
\hline bar 1,10 & 127,11 & 1,1 & 62,6 & .91 & .50 \\
\hline child 1,2 & 39,78 & 3,80 & 35,27 & .57 & .44 \\
\hline circuit $1,2,5$ & $67,6,7$ & $229,2,5$ & $23,2,8$ & .70 & .70 \\
\hline facility 1,4 & 26,61 & 4,18 & 15,28 & .79 & .67 \\
\hline grip 2,7 & 6,1 & 17,6 & 4,0 & 1.00 & 1.00 \\
\hline holiday 1,2 & 4,57 & 5,17 & 26,2 & .96 & .96 \\
\hline material 1,4 & 65,7 & 63,10 & 30,9 & .79 & .79 \\
\hline post $2,3,4,7,8$ & $1,64,20,11,7$ & $2,7,1,9,3$ & $2,25,13,12,4$ & .45 & .25 \\
\hline restraint $1,4,6$ & $17,32,11$ & $2,2,2$ & $8,14,4$ & .65 & .50 \\
\hline stress 1,2 & 3,45 & 8,50 & 1,19 & .95 & .95 \\
\hline Total & 773 & 547 & 379 & .73 & .58 \\
\hline
\end{tabular}

The most similar experiment in the literature is Agirre and Martínez (2000), in which the sense-tagged instances obtained using a high-performance Web-mining algorithm (Mihalcea and Moldovan 1999) performed hardly better than a random baseline as WSD training instances. A difference between the two experiments is that Agirre et al. do not limit their experiments to the fraction of the test set for which they have automatically extracted training samples; hence a direct comparison of the results is not possible.

A detailed examination of the results indicates that the difference in performance is related to the smaller number of training instances rather than to the quality of individual instances:

- In all four cases in which ODP provides a comparable-or larger-number of training instances (circuit, grip, material, stress), ODP training equals hand-tagged training. In one additional case (holiday), the number of ODP instances is smaller, but still the recall is the same. For the other five words, the number of ODP instances is substantially smaller and the recall is worse.

- Remarkably, incorrect directories harm recall substantially only for post, which accumulates six erroneous associations (out of 11 errors). The other five errors (in material 1, 4, holiday 2, grip 7) do not affect the final recall for these words. There are two possible reasons for this behavior:

- Erroneous directories tend to be less productive in terms of training instances. Indeed, this fact could be incorporated as an additional filter for candidate directories. This is the case, for instance, of material 1, for which correct directories provide much more training material than the incorrect one.

- $\quad$ Erroneous directories are more frequent with rare (less frequent) word senses. This is correlated with a smaller number of test instances (hence the influence on average recall is lower) and also of training instances (and then the reference, hand-tagged material does not provide good training data either). This is the 
case of grip 7 or holiday 2, which have zero and two test instances, respectively.

Overall, our results suggest that directory-based instances, in spite of being shorter and automatically extracted, are not substantially worse for supervised WSD than the hand-tagged material provided by the Senseval organization. The limitation of the approach is currently the low coverage of word senses and the amount of training samples. Two strategies may help in overcoming such limitations: first, propagating directories via synonymy (attaching directories to synsets rather than word senses) and semantic relationships (propagating directories via hyponymy relations); second, retrieving instances not only from the ODP page describing the directory contents, but from the Web pages listed in the directory.

The only fundamental limitation of our approach for the automatic extraction of annotated examples is the fact that directories are closely related to topics and domains, and therefore word senses that do not pertain to any domain cannot receive directories and training instances from them. Still, the approach can be very useful for language engineering applications in which only domain disambiguation (versus sense disambiguation) is required, such as information retrieval (Gonzalo et al. 1998) and content-based user modeling (Magnini and Strapparava 2000).

\section{Massive Processing of WordNet Nouns}

We have applied the association algorithm to all noncompound nouns in WordNet without nonalphabetic characters (e.g., sea lion and 10 are not included in the bulk processing). The results can be seen in Table 10. Overall, the system associates at least one directory with 13,375 nouns ( $28 \%$ of the candidate set).

The most direct way of propagating directories in the WordNet structure is extending sense/directory associations to synset/directory relations (i.e., if a word sense receives a directory, then all word senses in the same synset receive the same directory). For instance, cable 2 (transmission line) receives the following directories:

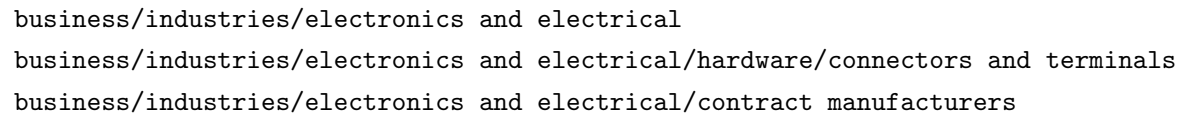

As cable 2 is part of the synset \{cable 2, line 9, transmission line 1\}, line 9 and transmission line 1 inherit the three directories.

With this (quite conservative) strategy, the number of characterized nouns and word senses almost doubles: 24,558 nouns and 27,383 senses, covering $34 \%$ of the can-

Table 10

Massive association of ODP directories with WordNet 1.7 nouns.

\begin{tabular}{lrc}
\hline & & With Propagation \\
Candidate nouns & 51,168 & \\
Candidate senses & 73,612 & \\
Associated directories & 29,291 & \\
Characterized nouns & 13,375 & 24,558 \\
Characterized senses & 14,483 & 27,383 \\
Hyponyms & 1,800 &
\end{tabular}


didate nouns plus 7,027 multiword terms that were not in the candidate set. The results of this massive processing, together with the results for the Senseval 2 test (including training material) are available for public inspection at $\langle$ http://nlp.uned.es/ODP $\rangle$.

\section{Conclusions}

Our algorithm is able to associate ODP directories with WordNet senses with $86 \%$ accuracy over the Senseval 2 test, and with coverage between $73 \%$ and $88 \%$ of the domain-specific senses. Such associations can be used as rich characterizations for word senses: as a source of information to cluster senses according to their topical relatedness, to extract topic signatures, to acquire sense-tagged corpora, etc. The only intrinsic limitation of the approach is that Web directories are not appropriate for characterizing general word senses (versus domain-specific ones). If such characterization is necessary for a particular natural language application, the method should be complemented by other means of acquiring lexical information.

In the supervised WSD experiment we have carried out, the results suggest that the characterization of word senses with Web directories provides cleaner data, without further sophisticated filtering, than a direct use of the full Web. Indeed the WSD results using training material from ODP directories gives better results than could be expected from previous cross-validations of training and test WSD materials.

Our ongoing work is extending the algorithm - which works independently for every input word-to combine and propagate sense/directory associations over the entire WordNet. The initial coverage of WordNet nouns is $34 \%$, but we hope to improve this figure by taking advantage of the WordNet structure.

Perhaps the main conclusion of our work is that Web directories are a much more structured and reliable corpus than the whole Web. In spite of being manually supervised, Web directories offer immense structured corpora that deserve our attention as sources of linguistic information. In particular, listing word sense/ODP directory associations has the additional advantage, compared to other Web-mining approaches, of providing a wealth of lexical information in a very condensed manner.

\section{Acknowledgments}

This work has been partially supported by the Spanish government through project Hermes (TIC2000-0335-C03-01).

\section{References}

Agirre, E., O. Ansa, E. Hovy, and D. Martínez. 2000. Enriching very large ontologies using the WWW. In Proceedings of the Ontology Learning Workshop, Berlin.

Agirre, E. and D. Martínez. 2000. Exploring automatic word sense disambiguation with decision lists and the Web. In Proceedings of the COLING Workshop on Semantic Annotation and Intelligent Content, Luxembourg.

Edmonds, P. and S. Cotton. 2001. Senseval-2: Overview. In Proceedings of Senseval 2. Association for Computational Linguistics, New Brunswick, NJ.

Fujii, A. and T. Ishikawa. 1999. Utilizing the World Wide Web as an encyclopedia:
Extracting term descriptions from semi-structured texts. In Proceedings of ACL-99. Association for Computational Linguistics, New Brunswick, NJ.

Gonzalo, J., F. Verdejo, I. Chugur, and J. Cigarrán. 1998. Indexing with Wordnet synsets can improve text retrieval. In COLING/ACL'98 Workshop on Usage of WordNet in Natural Language Processing Systems. Association for Computational Linguistics, New Brunswick, NJ.

Grefenstette, G. 1999. The WWW as a resource for example-based MT tasks. In Proceedings of ASLIB-99, London.

Joho, H. and M. Sanderson. 2000. Retrieving descriptive phrases from large amounts of free text. In Proceedings of the 9th ACM CIKM Conference, McLean, VA.

Kilgarriff, A. 2001a. English lexical sample task description. In Proceedings of Senseval 2. Association for Computational Linguistics, New Brunswick, NJ.

Kilgarriff, A. 2001b. Web as corpus. In 
Proceedings of Corpus Linguistics 2001, Lancaster, England.

Kilgarriff, A. and M. Palmer. 2000. Introduction to the special issue on Senseval. Computers and the Humanities, 34(1-2).

Ma, X. and M. Liberman. 1999. Bits: A method for bilingual text search over the Web. In Proceedings of the Machine Translation Summit VII, Singapore.

Magnini, B. and G. Cavaglia. 2000. Integrating subject field codes into WordNet. In Proceedings of LREC-2000, Second International Conference on Language Resources and Evaluation, Athens.

Magnini, B. and C. Strapparava. 2000. Experiments in word domain disambiguation for parallel texts. In ACL-2000 Workshop on Word Sense and Multilinguality. Association for Computational Linguistics, New Brunswick, NJ.

Mihalcea, R. and D. Moldovan. 1999a. An automatic method for generating sense tagged corpora. In Proceedings of AAAI '99, Orlando, FL, July, pages 461-466.

Miller, G. 1990. Wordnet: An on-line lexical database. Special issue. International Journal of Lexicography, 3(4).

Nie, Jian-Yun, Michel Simard, and George
Foster. 2001. Multilingual information retrieval based on parallel texts from the Web. In Carol Peters, Editor,

Cross-Language Information Retrieval and Evaluation: Workshop of Cross-Language Evaluation Forum (CLEF 2000), Lisbon, Portugal, September 21-22, 2000, Revised Papers. Lecture Notes in Computer Science 2069. Berlin, Springer-Verlag, pages $188-200$.

Pedersen, T. 2001. Machine Learning with lexical features: The Duluth approach to Senseval-2. In Proceedings of Senseval-2. Association for Computational Linguistics, New Brunswick, NJ.

Peters, C., M. Braschler, J. Gonzalo, and M. Kluck, editors. 2002. Evaluation of Cross-Language Information Retrieval Systems. Lecture Notes in Computer Science 2406. Springer-Verlag.

Resnik, P. 1999. Mining the Web for bilingual text. In Proceedings of the 37th Annual Meeting of the Association for Computational Linguistics, College Park, MD.

Resnik, P. and N. Smith. 2002. The Web as a parallel corpus. Technical Report UMIACS-TR-2002, University of Maryland. 